\title{
Perfil antropométrico da população idosa brasileira. Resultados da Pesquisa Nacional sobre Saúde e Nutrição
}

\author{
Anthropometric profile of the elderly Brazilian \\ population: results of the National Health and \\ Nutrition Survey, 1989
}

Elda Lima Tavares 1,2

Luiz Antonio dos Anjos 3,4

\footnotetext{
1 Secretaria Municipal de Saúde, Prefeitura do Rio de Janeiro. Rua Afonso Cavalcanti 455, sala 823, Cidade Nova, Rio de Janeiro, $R J$ 20211-110, Brasil. 2 Departamento de Nutrição Social, Instituto de Nutrição, Universidade do Estado do Rio de Janeiro. Rua São Francisco Xavier 524, 12 o andar, bloco D, sala 12001, Maracanã Rio de Janeiro, $R J$ 20555-013, Brasil. 3 Centro de Estudos da Saúde do Trabalhador e Ecologia Humana, Escola Nacional de Saúde Pública, Fundação Oswaldo Cruz. Rua Leopoldo Bulhões 1480, Manguinhos, Rio de Janeiro, RJ, 21045-900, Brasil. anjos@ensp.fiocruz.br 4 Laboratório de Avaliação Nutricional e Funcional, Departamento de Nutrição Social, Faculdade de Nutrição, Universidade Federal Fluminense. Rua São Paulo 30, 4o andar, Centro, Niterói, RJ 24015-110, Brasil.
}

\begin{abstract}
This paper presents the nutritional status of the elderly Brazilian population (60 years and older) based on a probabilistic sample survey in 1989. Nutritional assessment used the BMI ( $\mathrm{kg} / \mathrm{m} 2)$, with cut-off points recommended by the WHO (1995). Socio-demographic variables included age, gender, region and place of residence in the country (urban/rural area), income, education, and housing conditions. Prevalence rates for thinness (BMI < 18.5) and overweight (BMI $\geq$ 25) were $7.8 \%$ and $30.4 \%$ in men and $8.4 \%$ and $50.2 \%$ in women, higher than in the young adult population. Thinness was more frequent in older women, in rural areas from the Midwest/Northeast regions (women) and Southeast/Midwest (men), in groups with lower income, less schooling, and worse housing conditions. Overweight was more frequent in women from urban areas in the South and Southeast and groups with higher income, more schooling, and better housing conditions. The results indicate problematic nutritional status in the Brazilian elderly, particularly women.
\end{abstract}

Key words Anthropometry; Nutrition Surveys; Demographic Aging; Nutrition; Epidemiology

Resumo O presente artigo descreve o perfil nutricional da população idosa brasileira (idade igual ou superior a sessenta anos), com base nos dados da Pesquisa Nacional sobre Saúde e Nutrição, estudo com amostra probabilística realizado em 1989. Utilizaram-se para tal análise: o índice de massa corporal - IMC $\left(\mathrm{kg} / \mathrm{m}^{2}\right)$, como indicador do estado nutricional segundo recomendação da OMS (WHO, 1995), e as variáveis idade, gênero, região e situação do domicílio no país (urbana/rural), renda, escolaridade e qualidade de moradia. As prevalências gerais de magreza $(I M C<18,5)$ e sobrepeso (IMC $\geq 25)$ foram, respectivamente, 7,8\% e 30,4\% em homens e $8,4 \%$ e 50,2\% em mulheres, mais elevadas que na população adulta jovem. A magreza foi mais freqüente em mulheres nas faixas etárias avançadas; em áreas rurais das regiões Centro-Oestel Nordeste (mulheres) e Sudeste/Centro-Oeste (homens); nas classes de menor renda, menor escolaridade e pior qualidade de moradia. O sobrepeso foi prevalente em mulheres, em áreas urbanas das regiões Sul e Sudeste (ambos os sexos); nos grupos de maior renda, maior escolaridade e melhor qualidade de moradia. Os resultados indicam um quadro preocupante de alterações nutricionais em idosos no Brasil, principalmente em mulheres.

Palavras-chave Antropometria; Inquéritos Nutricionais; Envelhecimento da População; $\mathrm{Nu}$ trição; Epidemiologia 


\section{Introdução}

De acordo com as previsões da Organização Mundial de Saúde (OMS), no ano 2000 haverá 600 milhões de indivíduos idosos no mundo, dos quais dois terços em países em desenvolvimento (WHO, 1989). O Brasil destaca-se por apresentar uma das maiores taxas de crescimento desta população, com projeções para 2025 de cerca de 32 milhões de pessoas idosas (Kalache et al., 1987). A questão da terceira idade emerge como problema para investigação e planejamento de políticas públicas, em razão do inevitável crescimento de demanda ao sistema de saúde (Veras et al., 1987).

A nutrição é um aspecto importante neste contexto pela modulação das mudanças fisiológicas relacionadas com a idade e no desenvolvimento de doenças crônicas não-transmissíveis, como doenças cardiovasculares, diabetes, obesidade, osteoporose e alguns tipos de câncer (Dirren, 1994). Em idosos, no entanto, torna-se mais complexa a análise do estado nutricional em virtude da maior heterogeneidade entre os indivíduos deste grupo (Goodwin, 1989; WHO, 1989) e do fato de seu valor preditivo estar atrelado a um conjunto de fatores não apenas relacionado às mudanças biológicas da idade, doenças e mudanças seculares, como também às práticas ao longo da vida (fumo, dieta, atividade física) e aos fatores sócio-econômicos (WHO, 1995).

Deste modo, torna-se necessário produzir informações nutricionais referentes ao grupo para começar a avaliar sua problemática específica e enfrentar os desafios da pesquisa no campo de nutrição e envelhecimento dentro das peculiaridades do país. Assim, o presente estudo objetiva analisar a situação nutricional da população idosa brasileira, segundo variáveis sócio-demográficas, com base nos dados da Pesquisa Nacional sobre Saúde e Nutrição (PNSN).

\section{Metodologia}

A Pesquisa Nacional sobre Saúde e Nutrição (PNSN) foi conduzida entre junho e setembro de 1989, com o objetivo de aferir o estado nutricional da população brasileira, mediante coleta de dados antropométricos e outras informações sobre saúde, condições de vida e ocupação. O levantamento dos dados da PNSN foi patrocinado pelo Instituto Nacional de Alimentação e Nutrição (INAN) e executado em colaboração técnica da Fundação Instituto Brasileiro de Geografia e Estatística (IBGE) e do
Instituto de Pesquisas Econômicas Aplicadas (IPEA). As análises e interpretações apresentadas no presente trabalho são de inteira responsabilidade dos autores e não indicam aprovação das instituições acima referidas.

O estudo foi do tipo transversal (survey), de base domiciliar, excluindo-se as populações indígenas, de rua e institucionalizadas. Deste modo, deve-se ter cautela com análises nos grupos de idade mais avançada por causa dos possíveis efeitos: a) confundimento, em conseqüência da tendência secular, mortalidade e sobrevivência seletivas; b) viés, pelo fato da população idosa provavelmente apresentar uma proporção maior de pessoas vivendo em instituições, o que pode influenciar o estado nutricional do grupo.

A amostra da pesquisa foi estratificada, probabilística, com duas etapas de seleção (Fletcher, 1987). Na primeira etapa, definiram-se os âmbitos da pesquisa, que constaram das cinco macroregiões do país e das duas situações (urbana e rural), excluído o Norte rural, por dificuldades operacionais, atingindo-se no total nove âmbitos de análise. Cada âmbito da pesquisa tornou-se um estrato da amostra, no qual procedeu-se seleção referente aos setores censitários. Estes foram ordenados em três níveis sócio-econômicos, segundo renda domiciliar mensal média e taxa de alfabetização na faixa de 5 a 14 anos de idade, de acordo com informações do censo de 1980 projetadas para a população de 1989. Dezoito setores censitários de cada nível sócio-econômico foram sorteados por âmbito, totalizando assim 54 setores em cada âmbito e 486 em todo o país. Cada setor censitário foi considerado como unidade primária de seleção ou aglomerado da amostra, selecionando-se, na segunda etapa, uma média de 36 domicílios para entrevista em cada aglomerado, o que correspondeu ao total de 14.455 domicílios investigados. Todas as pessoas dos domicílios sorteados participaram da pesquisa, o equivalente a 63.213 pessoas pesquisadas, de todas as idades, sem exceção, residentes em 363 municípios brasileiros (Fletcher, 1987).

A casuística do presente estudo incluiu todos os indivíduos participantes da pesquisa e não-portadores de deficiências físicas que comprometessem os dados antropométricos e que tivessem sessenta anos de idade ou mais, que é o ponto de corte para definir idosos preconizado por instituições internacionais (WHO, 1989). Todas as análises foram expandidas para a população idosa brasileira, utilizando-se o fator de expansão da própria PNSN (PNSN-P), referente ao registro de pessoa, ou seja, o in- 
verso da probabilidade de seleção de cada pessoa no setor censitário (INAN, 1990).

Os valores da população brasileira expandida a partir da amostra não se diferenciaram muito das informações censitárias, sugerindose que a pesquisa foi representativa da realidade demográfica do país, próxima à época do estudo. Encontrou-se uma diferença na região Norte que pode ser resultante de subestimação verificada no processo de amostragem da população projetada pelo modelo demográfico do IBGE (INAN, 1990).

Os dados antropométricos coletados foram massa corporal e estatura. A massa corporal foi aferida em balança microeletrônica portátil, de marca nacional Filizola, com precisão de 0,1 kg e capacidade de $150 \mathrm{~kg}$, e a estatura, com fita métrica de precisão de $0,1 \mathrm{~cm}$. Utilizou-se para a análise antropométrica do estado nutricional: o Índice de Massa Corporal (IMC), calculado a partir da divisão da massa corporal em quilogramas pela estatura em metro elevado ao quadrado $\left(\mathrm{kg} / \mathrm{m}^{2}\right)$; e os pontos de corte recomendados pela Organização Mundial de Saúde (OMS) para avaliação de população adulta e idosa (WHO, 1995) - magreza severa (IMC < $16,0)$, magreza moderada $(16,0 \leq \mathrm{IMC}<17,0)$, magreza leve $(17,0 \leq$ IMC $<18,5)$, adequado $(18,5 \leq \mathrm{IMC}<25,0)$, sobrepeso grau I $(25,0 \leq$ IMC $<30,0)$, sobrepeso grau II $(30,0 \leq$ IMC $<$ 40,0 ) e sobrepeso grau III (IMC $\geq 40,0$ ).

As variáveis demográficas e sociais estudadas compreenderam as seguintes características:

a) Região e situação do domicílio no país (área urbana ou rural);

b) Gênero;

c) Faixa etária em intervalos de 5 anos;

d) Quartis de renda mensal domiciliar per capita em dólar, correspondentes aos seguintes valores médios na população à época do estudo: primeiro quartil, US\$ 12,7; segundo quartil, US\$ 31,1 ; terceiro quartil, US\$ 65,4; e o quarto quartil, US\$ 311,9 ;

e) Escolaridade, expressa pela capacidade de ler e escrever e os anos de estudo cursados, com seis categorias: escolaridade zero/analfabeto; escolaridade zero, mas com capacidade de ler e escrever; um a quatro anos de estudo; cinco a oito; nove a 11; e 12 anos ou mais;

f) Condições de moradia expressas pelo indicador proposto por Bronfman et al. (1988) e adaptado para a PNSN por Ribeiro (1994).

Este último conjuga variáveis relacionadas às características sócio-ambientais do domicílio, partindo-se das informações de piso do domicílio, qualidade do abastecimento de água, saneamento e aglomeração (pessoa/dormitó- rio), divididas em atributos, cujas classificações distribuídas de forma associada resultaram em três categorias finais para o indicador de moradia: bom, regular e mau.

Os dados foram processados com o programa estatístico SAS e analisados através da descrição bivariada de freqüências relativas do estado nutricional segundo as variáveis sócio-demográficas citadas.

\section{Resultados}

A população maior de sessenta anos na amostra da PNSN foi de 4.277 indivíduos, correspondente em números expandidos a mais de dez milhões de idosos, cerca de $7,2 \%$ da população total estimada do país. Do total de idosos, $2.028(47,4 \%)$ eram do sexo masculino e $2.249(52,6 \%)$ do sexo feminino, com a predominância do sexo feminino em praticamente todas as faixas etárias. Em ambos os sexos, pouco mais de $60 \%$ encontrava-se no intervalo de 60 a 69 anos, e $90 \%$ desta população estava abaixo de oitenta anos.

A distribuição regional da população idosa apresentou a seguinte ordem: Sudeste e Nordeste em primeiro e segundo lugares, com as maiores proporções, seguidos pelo Sul, CentroOeste e, por último, a região Norte. Observouse predomínio de idosos residentes em área urbana, havendo no país maior proporção de mulheres $(77 \%)$ do que homens (68\%) vivendo nessa situação.

Em homens idosos, a prevalência de magreza geral $(7,8 \%)$ foi maior que os sobrepesos II e III $(5,7 \%)$, enquanto no grupo feminino ocorreu o contrário, 8,4\% contra 18,2\% (Tabela 1). A freqüência de magreza, inclusive as mais intensas, aumentaram nas últimas faixas de idade, com ligeira predominância em mulheres, quando comparadas aos homens até a faixa de 70 a 75 anos. Encontrou-se uma prevalência de sobrepeso geral de 30,4\% em homens e de $50,2 \%$ em mulheres, predominando esse problema no sexo feminino em todos os graus. Observou-se menor proporção de sobrepeso nos grupos de idade mais avançada em ambos os sexos, não ocorrendo os casos mais graves em homens acima de setenta anos e mulheres acima de oitenta anos.

Verificou-se uma tendência de maiores proporções de magreza e sobrepeso na população idosa em comparação com adultos na faixa de vinte a quarenta anos. A prevalência de magreza em homens idosos $(7,8 \%)$ foi cerca de 2,2 vezes maior do que a encontrada entre os mais jovens (3,6\%); e, em mulheres idosas $(8,4 \%), 1,2$ 
Distribuição percentual de estado nutricional na população idosa brasileira (idade $\geq 60$ anos), segundo Índice de Massa

Corporal (IMC)*, por sexo e faixa etária, Pesquisa Nacional sobre Saúde e Nutrição, 1989.

\begin{tabular}{|c|c|c|c|c|c|c|c|c|c|}
\hline \multirow[t]{2}{*}{ Idade (anos) } & \multirow[t]{2}{*}{$\begin{array}{l}\text { Número } \\
\text { amostral }\end{array}$} & \multirow[t]{2}{*}{$\begin{array}{l}\text { Número } \\
\text { expandido }\end{array}$} & \multicolumn{7}{|c|}{ Estado Nutricional $(\%)^{\star \star}$} \\
\hline & & & Severa & Moderada & Leve & & Grau I & Grau II & Grau III \\
\hline \multicolumn{10}{|l|}{ Homens } \\
\hline $60-64,9$ & 723 & 1.639 .264 & 0,4 & 1,0 & 3,6 & 61,0 & 27,2 & 6,4 & 0,4 \\
\hline $65-69,9$ & 518 & 1.150 .198 & 0,4 & 0,8 & 6,8 & 59,9 & 25,9 & 5,9 & 0,3 \\
\hline $70-74,9$ & 371 & 858.116 & 1,2 & 1,8 & 4,5 & 63,4 & 24,5 & 4,6 & 0,0 \\
\hline $75-79,9$ & 239 & 481.865 & 3,4 & 4,6 & 9,0 & 58,9 & 17,1 & 7,0 & 0,0 \\
\hline 80 e + & 177 & 407.181 & 1,6 & 0,5 & 6,4 & 70,5 & 19,8 & 1,2 & 0,0 \\
\hline Total & 2.028 & 4.536 .624 & 1,0 & 1,4 & 5,4 & 61,8 & 24,7 & 5,5 & 0,2 \\
\hline \multicolumn{10}{|l|}{ Mulheres } \\
\hline $60-64,9$ & 787 & 1.986 .190 & 0,6 & 0,9 & 2,1 & 45,3 & 32,6 & 18,3 & 0,2 \\
\hline $65-69,9$ & 571 & 1.450 .602 & 1,6 & 2,3 & 5,5 & 32,9 & 34,6 & 21,6 & 1,5 \\
\hline $70-74,9$ & 388 & 1.018 .295 & 3,0 & 2,2 & 5,3 & 41,9 & 30,4 & 16,2 & 1,0 \\
\hline $75-79,9$ & 290 & 677.489 & 3,3 & 2,0 & 8,7 & 39,0 & 30,6 & 14,6 & 1,8 \\
\hline 80 e + & 213 & 560.203 & 1,2 & 1,7 & 8,8 & 51,8 & 28,2 & 8,3 & 0,0 \\
\hline Total & 2.249 & 5.692 .779 & 1,7 & 1,7 & 5,0 & 41,4 & 32,0 & 17,3 & 0,9 \\
\hline
\end{tabular}

* $\quad \mathrm{IMC}=\mathrm{kg} / \mathrm{m}^{2}$

** Magreza severa (IMC<16,0); magreza moderada $(16,0 \leq \mathrm{IMC}<17,0)$; magreza leve $(17,0 \leq \mathrm{IMC}<18,5)$; adequado $(18,5 \leq \mathrm{IMC}<25,0)$; sobrepeso I $(25,0 \leq$ IMC $<30,0)$, sobrepeso II e III $($ IMC $\geq 30,0)$

vezes maior em comparação com as jovens (6,9\%). Quanto ao sobrepeso, verificou-se uma freqüência 1,5 vezes maior de sobrepeso II e III em homens idosos $(5,7 \%)$ em relação aos jovens $(3,7 \%)$ e 2,5 vezes mais em mulheres idosas $(18,2 \%)$ quando comparadas às jovens $(7,2 \%)$.

As alterações nutricionais observadas apresentaram grande variação entre as regiões do país (Tabelas 2 e 3). As maiores prevalências de magreza em homens foram encontradas nas regiões Centro-Oeste $(10,7 \%)$, Nordeste $(8,8 \%)$ e Sudeste (7,9\%); e, em mulheres, no Nordeste $(11,9 \%)$, Centro-Oeste $(11,6 \%)$ e Norte $(9,6 \%)$. Estas foram superiores em mulheres em todas as regiões, com exceção do Sudeste. O sobrepeso foi mais freqüente nas regiões $\mathrm{Sul}$ e $\mathrm{Su}-$ deste, encontrando-se 9,2\% e 6,4\% dos idosos e $21,9 \%$ das idosas das respectivas regiões classificados nos graus II e III.

Confirmou-se a predominância de magreza nas áreas rurais de todas as regiões para ambos os sexos, exceto para o grupo de mulheres da região Sul, que apresentou uma prevalência menor na área rural quando comparada à urbana (Tabela 3). No grupo de idosos, a pior situação encontrou-se no Sudeste $(17,3 \%)$ e Centro-Oeste $(11,0 \%)$ rural, destacando-se também alta prevalência no Centro-Oeste ur- bano (10,6\%). As maiores freqüências de incidência de magreza em idosas foram nas de áreas rurais do Centro-Oeste (18,6\%) e Nordeste $(17,6 \%)$, apresentando-se as áreas urbanas destas regiões valores relevantes (quase 10\%).

O sobrepeso foi mais prevalente nas áreas urbanas de todas as regiões para homens e mulheres em todos os graus. O Sul e Sudeste urbanos apresentaram as maiores prevalências de sobrepesos II e III em idosos (11,3\% e 7,8\%) e idosas (23,2\% e 23,3\%), com exceção da região Sul, onde houve uma freqüência pouco maior em mulheres da área rural $(23,7 \%)$.

Segundo a distribuição das variáveis sociais apresentadas nas Tabelas 4 e 5, encontrou-se no primeiro quartil de renda, grupo de maior pobreza, $11,2 \%$ dos homens e $13,9 \%$ das mulheres com magreza. No quarto quartil, grupo de maior nível de renda, apenas 3,6\% e 2,7\% apresentavam este problema, respectivamente. O inverso aconteceu nos sobrepesos II e III, pois sua prevalência passou de 1,4\% em homens e $12,1 \%$ em mulheres do primeiro quartil para $10,5 \%$ e $22,1 \%$ nos respectivos grupos do último quartil.

Na categoria de analfabetismo, cerca de $13 \%$ dos idosos (homens e mulheres) apresentavam magreza, reduzindo-se a zero sua freqüência no grupo com 12 anos ou mais de es- 
Distribuição percentual do estado nutricional em homens idosos brasileiros (idade $\geq 60$ anos), segundo Índice de Massa Corporal (IMC)*, por macroregiões e situação de domicílio, Pesquisa Nacional sobre Saúde e Nutrição, 1989.

\begin{tabular}{|c|c|c|c|c|c|}
\hline \multirow[t]{2}{*}{ Regiões } & \multirow[t]{2}{*}{ Número } & \multicolumn{4}{|c|}{ Estado Nutricional $(\%)^{\star \star}$} \\
\hline & & Magreza & Adequado & Sobrepeso I & Sobrepeso II e II \\
\hline Norte & 223 & 4,4 & 60,6 & 29,4 & 5,6 \\
\hline Nordeste & 586 & 8,8 & 68,3 & 19,8 & 3,1 \\
\hline Urbano & 267 & 7,1 & 62,3 & 26,6 & 4,0 \\
\hline Rural & 319 & 10,7 & 74,6 & 12,5 & 2,2 \\
\hline Sudeste & 463 & 7,9 & 59,0 & 26,7 & 6,4 \\
\hline Urbano & 197 & 5,6 & 56,4 & 30,2 & 7,8 \\
\hline Rural & 266 & 17,3 & 69,5 & 12,4 & 0,8 \\
\hline Sul & 429 & 5,1 & 56,5 & 29,2 & 9,2 \\
\hline Urbano & 197 & 4,5 & 51,2 & 33,0 & 11,3 \\
\hline Rural & 232 & 6,4 & 66,4 & 22,0 & 5,2 \\
\hline Centro-Oeste & 327 & 10,7 & 60,6 & 22,8 & 5,9 \\
\hline Urbano & 154 & 10,6 & 55,2 & 27,3 & 6,9 \\
\hline Rural & 173 & 11,0 & 71,4 & 13,7 & 3,9 \\
\hline Brasil & 2.028 & 7,8 & 61,8 & 24,7 & 5,7 \\
\hline Urbano & 1.038 & 6,0 & 57,2 & 29,5 & 7,3 \\
\hline Rural & 990 & 11,7 & 71,7 & 14,2 & 2,4 \\
\hline
\end{tabular}

* $\mathrm{IMC}=\mathrm{kg} / \mathrm{m}^{2}$

** Magreza (todas as formas - IMC < 18,5); adequado $(18,5 \leq \mathrm{IMC}<25,0)$; sobrepeso I $(25,0 \leq \mathrm{IMC}<30,0)$, sobrepeso II e III (IMC $\geq 30,0)$.

colaridade. Por outro lado, observou-se um aumento da presença dos sobrepesos II e III, de $2 \%$ em homens e $13,4 \%$ em mulheres analfabetos para $14,6 \%$ e $31,2 \%$, respectivamente, nos que cursaram de 9 a 11 anos. A partir deste nível de escolaridade, parece haver uma estabilização em homens e reversão do sobrepeso em mulheres.

As maiores freqüências de magreza ocorreram nos grupos de piores condições de moradia (12,5\% em homens e 15,9\% em mulheres), enquanto que a ocorrência nos que se encontravam em boas condições foi de 4,5\% e 4,4\%, respectivamente. Os sobrepesos II e III também apresentaram expressiva diferença de prevalências entre as classes extremas, variando de 1,5\% e 7,1\% (más condições) para 9,1\% e $23,2 \%$ (boas condições), em idosos e idosas.

\section{Discussão}

A proporção de idosos obtida pela expansão da amostra aproximou-se da encontrada no censo de 1991, 7,4\% da população brasileira, consti- tuindo-se este o grupo etário populacional de maior crescimento nas últimas décadas no país (Monteiro \& Alves, 1995). A predominância observada de mulheres confirma a maior expectativa de vida deste grupo, resultante do crescente excedente de mortalidade masculina em relação à feminina em todas as idades (Veras, 1994). Os dados confirmam o predomínio de uma população idosa jovem no Brasil, situada na faixa de 60 a 69 anos, $60 \%$, aspecto que influencia o seu perfil de saúde, visto que pessoas muito idosas são geralmente mais frágeis e demandam serviços de maior complexidade.

A distribuição demográfica da população idosa nas regiões do país reflete tanto os diferenciais de desenvolvimento sócio-econômico como os diferentes padrões de migração (Monteiro \& Alves, 1995). O processo de urbanização tem papel relevante nas questões relacionadas à saúde e nutrição, pois as diferentes condições de migração contribuem para a caracterização do grupo de idosos como não-homogêneo, resultante de diferentes exposições aos riscos do ambiente urbano (Solomons \& Mazariegos, 1992). 
Distribuição percentual do estado nutricional em mulheres idosas brasileiras (idade $\geq 60$ anos), segundo Índice de Massa Corporal (IMC)*, por macroregiões e situação de domicílio, Pesquisa Nacional sobre Saúde e Nutrição, 1989.

\begin{tabular}{|c|c|c|c|c|c|}
\hline \multirow[t]{2}{*}{ Regiões } & \multirow[t]{2}{*}{ Número } & \multicolumn{4}{|c|}{ Estado Nutricional (\%)** } \\
\hline & & Magreza & Adequado & Sobrepeso I & Sobrepeso II e II \\
\hline Norte & 266 & 9,6 & 43,9 & 33,4 & 13,1 \\
\hline Nordeste & 654 & 11,9 & 50,7 & 26,5 & 10,9 \\
\hline Urbano & 373 & 8,4 & 48,7 & 28,5 & 14,4 \\
\hline Rural & 281 & 17,6 & 54,0 & 23,1 & 5,3 \\
\hline Sudeste & 550 & 6,5 & 37,3 & 34,3 & 21,9 \\
\hline Urbano & 292 & 5,7 & 35,2 & 35,8 & 23,3 \\
\hline Rural & 258 & 11,2 & 50,8 & 24,5 & 13,5 \\
\hline Sul & 488 & 6,1 & 35,5 & 35,1 & 23,3 \\
\hline Urbano & 273 & 6,4 & 34,4 & 36,0 & 23,2 \\
\hline Rural & 215 & 5,3 & 38,7 & 32,3 & 23,7 \\
\hline Centro-Oeste & 291 & 11,6 & 42,6 & 34,4 & 11,4 \\
\hline Urbano & 169 & 9,3 & 41,7 & 37,0 & 12,0 \\
\hline Rural & 122 & 18,6 & 45,6 & 26,4 & 9,4 \\
\hline Brasil & 2.249 & 8,4 & 41,4 & 32,0 & 18,2 \\
\hline Urbano & 1.373 & 6,8 & 38,9 & 34,0 & 20,3 \\
\hline Rural & 876 & 13,7 & 50,0 & 25,3 & 11,0 \\
\hline
\end{tabular}

* $\mathrm{IMC}=\mathrm{kg} / \mathrm{m}^{2}$

** Magreza (todas as formas - IMC $<18,5)$; adequado $(18,5 \leq \mathrm{IMC}<25,0)$; sobrepeso I $(25,0 \leq \mathrm{IMC}<30,0)$, sobrepeso II e III (IMC $\geq 30,0)$.

A determinação do estado nutricional de idosos abrange uma complexa rede de fatores, além dos econômicos e alimentares, dentre os quais é possível citar o isolamento social e a solidão, as doenças crônicas e/ou incapacidades e as alterações fisiológicas do trato gastrointestinal decorrentes da idade (Solomons, 1992). Têm-se indicado o déficit energético-protéico como fator relacionado à imunossupressão e maior risco de infecções, importante causa de morbi-mortalidade em idosos (Chandra, 1990), e ao aumento de propensão a quedas (Vellas et al., 1990). A magreza excessiva nesta população tem sido apontada como fator mais fortemente associado à mortalidade do que o excesso de peso (Tayback et al., 1990).

O grupo feminino apresentou maiores prevalências de formas moderadas e severas de magreza em quase todas as faixas etárias e regiões do país, documentando um quadro de maior gravidade. Segundo o World Bank (1994), a má-nutrição crônica é comum entre as mulheres de países pobres e, em períodos de escassez alimentar, as idosas são as mais afetadas adversamente pela subnutrição. Os proble- mas de pobreza na velhice são, em parte, um problema de mulheres, visto que estas predominam entre os muito idosos, e os que têm pouca saúde têm rendimentos mais exíguos e padecem mais de solidão (Veras, 1994).

$\mathrm{O}$ fato dessas freqüências aumentarem nos grupos de maior faixa etária em ambos os sexos pode estar refletindo diferenças geracionais, aspectos de mortalidade ou sobrevivência seletivas. No entanto, a tendência de redução de massa corporal média de populações a partir de certa idade (Anjos, 1993), principalmente de massa corporal magra (Forbes \& Reina, 1970) e água corporal (Steen, 1988), tem sido verificada também em análises longitudinais.

A prevalência da magreza em idosos no Brasil, principalmente na área rural, apresenta-se preocupante por estar dentro de limites $(10 \%$ a $19 \%$ ) referidos pela OMS como marcadores de situação de pobreza para a população adulta (WHO, 1995). O grupo masculino apresentou maior freqüência no Sudeste rural, e o feminino, no Centro-Oeste rural, podendo-se especular que essas ocorrências se relacionem com o processo de migração e condições sócio-eco- 
Distribuição percentual do estado nutricional em homens idosos brasileiros (idade $\geq 60$ anos), segundo Índice de Massa Corporal (IMC)*, por renda mensal domiciliar per capita, anos de escolaridade e condições de moradia, Pesquisa Nacional sobre Saúde e Nutrição, 1989.

\begin{tabular}{|c|c|c|c|c|c|}
\hline \multirow{2}{*}{ Regiões } & \multirow[t]{2}{*}{ Número } & \multicolumn{4}{|c|}{ Estado Nutricional $(\%)^{\star \star}$} \\
\hline & & Magreza & Adequado & Sobrepeso I & Sobrepeso II e II \\
\hline \multicolumn{6}{|c|}{$\begin{array}{l}\text { Renda domiciliar } \\
\text { per capita (quartil) }\end{array}$} \\
\hline $1 ㅇ$ & 397 & 11,3 & 75,3 & 12,2 & 1,2 \\
\hline 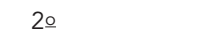 & 622 & 10,6 & 66,0 & 21,3 & 2,1 \\
\hline 3으 & 463 & 7,5 & 58,3 & 26,3 & 7,9 \\
\hline 4 으 & 443 & 3,5 & 49,1 & 36,8 & 10,6 \\
\hline Total & 1.925 & 8,0 & 61,1 & 25,1 & 5,8 \\
\hline \multicolumn{6}{|c|}{ Anos de escolaridade } \\
\hline 0 - analfabeto & 813 & 12,6 & 69,4 & 16,0 & 2,0 \\
\hline 0 - lê e escreve & 147 & 3,9 & 68,9 & 20,9 & 6,3 \\
\hline 1 a 4 & 777 & 6,8 & 58,7 & 28,3 & 6,2 \\
\hline 5 a 8 & 179 & 2,8 & 53,6 & 31,4 & 12,2 \\
\hline 9 a 11 & 61 & 0,3 & 43,5 & 41,6 & 14,6 \\
\hline $12 \mathrm{e}+$ & 43 & 0,0 & 41,9 & 48,4 & 9,7 \\
\hline Total & 2.020 & 7,9 & 61,7 & 24,7 & 5,7 \\
\hline \multicolumn{6}{|c|}{ Condições de moradia } \\
\hline Má & 670 & 12,5 & 75,3 & 10,7 & 1,5 \\
\hline Regular & 590 & 9,8 & 68,6 & 18,6 & 3,0 \\
\hline Boa & 759 & 4,5 & 51,9 & 34,5 & 9,1 \\
\hline Total & 2.019 & 7,8 & 61,8 & 24,7 & 5,7 \\
\hline
\end{tabular}

* $\quad \mathrm{IMC}=\mathrm{kg} / \mathrm{m}^{2}$

** Magreza (todas as formas - IMC $<18,5)$; adequado $(18,5 \leq \mathrm{IMC}<25,0)$; sobrepeso I $(25,0 \leq \mathrm{IMC}<30,0)$, sobrepeso II e III (IMC $\geq 30,0)$.

nômicas associadas, diferenciados por gênero nas diferentes regiões.

As desigualdades sociais são aspectos importantes no grupo de idosos, principalmente no espaço heterogêneo das grandes cidades (Veras, 1994). Em São Paulo, Ramos et al. (1993) encontraram que maior proporção de pessoas idosas residentes na periferia dispõe de menor renda, vive em domicílios multigeracionais e apresenta baixo nível de escolaridade.

Os resultados da PNSN indicam uma associação entre as precárias condições de vida e magreza na população idosa de forma geral. Observou-se que a elevação do nível de renda, do grau de escolaridade e da qualidade da moradia, relacionados a maior acesso a bens e serviços, reduziram, até mesmo eliminando, este problema nos grupos sociais mais privilegiados. A influência destas variáveis sobre o estado nutricional de idosos pode não ter relação igual à existente na população jovem, visto que a renda do indivíduo na velhice, por exemplo, pode estar bastante reduzida em comparação à sua própria renda no passado, em face da aposentadoria. De qualquer forma, o monitoramento populacional do estado nutricional é mais comum quando há risco de desnutrição aguda, e, nesse contexto, os idosos podem servir como sentinela para toda a população. Além disso, quando em população idosa os recursos são limitados, há necessidade de triagem para programas nutricionais e de saúde (WHO, 1995).

Quanto ao sobrepeso, sua tendência de aumento tem sido acompanhada em países desenvolvidos, questionando-se suas implicações na morbi-mortalidade futura, particularmente em populações que envelhecem (Kuczsmarski et al., 1994). Segundo Popkins (1994), esse processo estaria acontecendo atualmente nos países em desenvolvimento, nos quais os problemas de desnutrição e obesidade coexistem, observando-se aumento proporcional do consumo de dietas caracterizadas como de risco para doenças crônicas. Tem-se verificado na 
Distribuição percentual do estado nutricional em mulheres idosas brasileiras (idade $\geq 60$ anos), segundo Índice de Massa Corporal (IMC)*, por renda mensal domiciliar per capita, anos de escolaridade e condições de moradia, PNSN, 1989.

\begin{tabular}{|c|c|c|c|c|c|}
\hline \multirow[t]{2}{*}{ Regiões } & \multirow[t]{2}{*}{ Número } & \multicolumn{4}{|c|}{ Estado Nutricional $(\%)^{\star \star}$} \\
\hline & & Magreza & Adequado & Sobrepeso I & Sobrepeso II e II \\
\hline \multicolumn{6}{|l|}{$\begin{array}{l}\text { Renda domiciliar } \\
\text { per capita (quartil) }\end{array}$} \\
\hline $1 ㅇ$ & 421 & 13,8 & 48,0 & 26,0 & 12,2 \\
\hline 20 & 640 & 11,1 & 45,4 & 28,5 & 15,0 \\
\hline 3으 & 491 & 8,1 & 38,9 & 32,4 & 20,6 \\
\hline 4으 & 499 & 2,8 & 35,2 & 39,9 & 22,1 \\
\hline Total & 2.051 & 8,3 & 41,2 & 32,5 & 18,0 \\
\hline \multicolumn{6}{|c|}{ Anos de escolaridade } \\
\hline 0 - analfabeto & 1.112 & 13,0 & 45,4 & 28,2 & 13,4 \\
\hline 0 - lê e escreve & 128 & 7,3 & 43,2 & 25,5 & 24,0 \\
\hline 1 a 4 & 721 & 4,9 & 37,6 & 36,6 & 20,9 \\
\hline 5 a 8 & 199 & 1,9 & 40,6 & 34,6 & 22,9 \\
\hline 9 a 11 & 56 & 2,1 & 17,5 & 49,2 & 31,2 \\
\hline $12 e+$ & 29 & 0,0 & 60,1 & 25,2 & 14,7 \\
\hline Total & 2.245 & 8,4 & 41,5 & 32,0 & 18,1 \\
\hline \multicolumn{6}{|c|}{ Condições de moradia } \\
\hline Má & 598 & 15,9 & 56,3 & 20,7 & 7,1 \\
\hline Regular & 651 & 11,7 & 42,7 & 30,2 & 15,4 \\
\hline Boa & 994 & 4,4 & 35,8 & 36,6 & 23,2 \\
\hline Total & 2.243 & 8,3 & 41,4 & 32,1 & 18,2 \\
\hline
\end{tabular}

* $\mathrm{IMC}=\mathrm{kg} / \mathrm{m}^{2}$

** Magreza (todas as formas - IMC $<18,5)$; adequado $(18,5 \leq \mathrm{IMC}<25,0)$; sobrepeso I $(25,0 \leq \mathrm{IMC}<30,0)$, sobrepeso II e III (IMC $\geq 30,0)$.

população adulta brasileira expressivo aumento do sobrepeso nas últimas décadas, mais intenso justamente nas camadas sócio-econômicas intermediárias e baixas, situação que sinaliza a obesidade como questão de saúde pública e a necessidade de adoção de políticas preventivas (Monteiro et al., 1995).

Os resultados de sobrepeso geral e extremo em idosos confirmam a tendência encontrada em adultos de freqüências bastante elevadas em mulheres (Anjos, 1993). No estudo EURONUT-SENECA sobre o perfil nutricional e de saúde de idosos europeus foram encontradas variações de sobrepesos II e III entre 7\% (Noruega) e 43\% (Itália) em homens e 4\% (França) e 56\% (Itália) em mulheres (Dirren, 1994). A ocorrência desse problema em idosas brasileiras, principalmente das regiões Sul $(23,3 \%)$ e Sudeste $(21,9 \%$,) se aproxima a de países da Europa com altas prevalências.

O sobrepeso é um problema eminentemente urbano, com algumas diferenciações por região. Na região Sul, o excesso de peso em mu- lheres é também elevado na área rural. Tais resultados podem estar refletindo a presença diferenciada de fatores determinantes de sobrepeso relacionados a condições de vida, história ocupacional e estilos de vida, principalmente atividade física e consumo alimentar, conforme gênero, nas diversas realidades sociais e culturais brasileiras.

O sobrepeso apresentou uma associação positiva com o nível de renda, e esta foi mais intensa em homens. Tal comportamento pode sugerir duas reflexões que merecem maior aprofundamento: a) o aumento da renda como um fator mais forte na determinação do sobrepeso extremo masculino em virtude de mudanças importantes no estilo de vida (François \& James, 1994); b) a existência de grande parcela de população idosa feminina com altas proporções de sobrepeso já em estratos de baixa renda.

O impacto da escolaridade foi de aumento das prevalências de sobrepeso até a faixa de 9 a 11 anos cursados. Anjos (1993) observou na população adulta brasileira um aumento da 
mediana de massa corporal e IMC de homens paralelos ao aumento de renda e escolaridade. De forma contrária, a mediana de IMC encontrada em mulheres diminuiu a partir dos 5 anos de escolaridade em todos os estratos de renda, o que sugere a presença de elementos culturais relacionados ao corpo da mulher. Nas idosas, esta diminuição ocorreu acima de 12 anos de escolaridade, sugerindo, assim, uma influência de mudanças de comportamento frente ao sobrepeso na sociedade entre estas coortes.

$\mathrm{O}$ indicador de qualidade de moradia refletiu sensivelmente as mudanças no padrão nutricional desta população. Apesar deste índice não expressar necessariamente a história do grupo e dos aspectos ambientais, como, por exemplo, saneamento, não estarem tão diretamente associados à magreza em idosos como à desnutrição na criança, parece ser um bom marcador de condições gerais que acompanham diferentes arranjos e estilos de vida, perfis de morbidades e acesso a serviços de saúde da família e, por conseguinte, do idoso. Talvez o mesmo revele um retrato mais próximo das condições sócio-econômicas do grupo à época do estudo do que as variáveis renda domiciliar e escolaridade. As últimas podem ser influenciadas por padrões de distribuição intra-familiar de renda diferentes, conforme o fato do idoso morar só ou em domicílios multigeracionais, ou por questões culturais, como o menor acesso da mulher à escola em gerações passadas.

\section{Agradecimentos}

Aos professores Dr. Ricardo Ventura Santos e Dra. Rosely Sichieri pelas contribuições em versão anterior do presente trabalho.
Ainda não há consenso sobre o impacto do sobrepeso na longevidade, porém vêm-se encontrando associações deste problema com mortalidade em idosos (Harris et al., 1988; Cornoni-Huntley et al., 1991) e questionando-se sua relação com incapacidades (Rissanen et al., 1990), que nestes indivíduos significa autonomia, fator imprescindível para uma boa qualidade de vida. Além disso, as dificuldades relacionadas ao poder preditivo do IMC em idosos no diagnóstico da obesidade e do padrão de distribuição de gordura corporal (Kuczsmarski, 1989), bem como a falta de controle de algumas variáveis de confundimento, como, por exemplo, tabagismo, na maioria dos estudos (Manson et al., 1987), pode estar subestimando o impacto da obesidade nos resultados em saúde.

Em suma, a realidade brasileira requer estudos mais elaboradas e análises de tendências em vista da extrema heterogeneidade sócioeconômica do país que ainda pode ser forte determinante dos agravos nutricionais encontrados em idosos. As altas freqüências de magreza e sobrepeso revelam as contradições do perfil nutricional desta população, principalmente em mulheres. Como ambas alterações estão intimamente ligadas a doenças neste grupo, seja como fator de risco ou conseqüência, é importante aprofundar informações nutricionais para estabelecer práticas de monitoramento e direcionar intervenções mais adequadas a suas especificidades.

\section{Referências}

ANJOS, L. A., 1993. Valores Antropométricos da População Adulta Brasileira: Resultados da Pesquisa Nacional sobre Saúde e Nutrição. Tese apresentada ao Departamento de Nutrição Social da Universidade Federal Fluminense para concorrer à vaga de professor titular em Nutrição em Saúde Pública. Niterói: Universidade Federal Fluminense.

BRONFMAM, M.; GUISCAFRE, H. P.; CASTRO, R. \& GUTIERREZ, G., 1988. La medición de la desigualdad: Una estrategia metodológica, análisis de las características socioeconómicas de la muestra. Archivos de Investigación Médica, 19:351-360.

CHANDRA, R. K., 1990. The relation between immunology, nutrition and disease in elderly people. Age and Ageing, 19:525-531. 
CORNONI-HUNTLEY, J. C.; HARRIS, T. B.; EVERETT, D. F.; ALBANES, D.; MICOZZI, M. S.; MILES, T. P. \& FELDMAN, J. J., 1991. An overview of body weight of older persons, including the impact on mortality - The National Health and Nutrition Examination Survey I Epidemiologic follow-up study. Journal of Clinical Epidemiology, 44:743-753.

DIRREN, H. M., 1994. Euronut-SENECA: An european study of nutrition and health in the elderly. $\mathrm{Nu}$ trition Reviews, 52:S38-S43.

FLETCHER, P. R., 1987. Pesquisa Nacional sobre Saúde e Nutrição: Plano de Amostragem. Brasília: Organização Internacional do Trabalho/Programa das Nações Unidas para o Desenvolvimento/ Empresa de Informática e Planejamento/Instituto de Pesquisas Econômicas Aplicadas. (mimeo.)

FORBES, G. B. \& REINA, J. C., 1970. Adult lean body mass declines with age: Some longitudinal observations. Metabolism, 19:653-663.

FRANCOOIS, P. J. \& JAMES, W. P. T., 1994. An assessment of nutritional factors affecting the BMI of a population. European Journal of Clinical Nutrition, 48:S110-S114.

GOODWIN, J. S., 1989. Social, psychological and physical factors affecting the nutrition status of elderly subjects: Separating cause and effect. American Journal of Clinical Nutrition, 50:12011209.

HARRIS, T.; COOK, E. F.; GARRISON, R.; HIGGINS, M.; KANNEL, W. \& GOLDMAN, L., 1988. Body Mass Index and mortality among nonsmoking older persons: The Framingham Heart Study. JAMA, 259:1529-1534.

INAN (Instituto Nacional de Alimentação e Nutrição), 1990. Pesquisa Nacional sobre Saúde e Nutrição, PNSN, 1989 - Arquivo de Dados da Pesquisa. Brasília: INAN/Ministério da Saúde. (mimeo.)

KALACHE, A.; VERAS, R. P. \& RAMOS, L. R., 1987. O envelhecimento da população mundial. Um desafio novo. Revista de Saúde Pública, 21:200-210.

KUCZMARSKI, R. J., 1989. Need for body composition in elderly subjects. American Journal of Clinical Nutrition, 50:1150-1157.

KUCZMARSKI, R. J.; FLEGAL, K. M.; CAMPBELL, S. M. \& JOHNSON, C. L., 1994. Increasing prevalence of overweight among US adults: The National Health and Nutrition Examination Surveys, 1960 to 1991. Journal of The American Medical Association, 272:205-211.

MANSON, J. E.; STAMPFER, M. J.; HENNEKENS, C. H. \& WILLLET, W. C., 1987. Body weight and longevity. Journal of The American Medical Association, 257:353-358.

MONTEIRO, M. M. G. \& ALVES, M. I. C., 1995. Aspectos demográficos da população idosa no Brasil. In:. Terceira Idade: Um Envelhecimento Digno para o Cidadão do Futuro (R. Veras, org.), pp. 6578, Rio de Janeiro: Editora Relume-Dumará/Universidade Aberta da Terceira Idade - UNATI, Universidade do Estado do Rio de Janeiro.
MONTEIRO, C. A.; MONDINI, L.; SOUZA, A. L. M. \& POPKINS, B. M., 1995. Da desnutrição para a obesidade: A transição nutricional no Brasil. In: Velhos e Novos Males da Saúde no Brasil: A Evolução do País e de suas Doenças (C. A. Monteiro, org.), pp. 247-255, São Paulo: Editora Hucitec/ Núcleo de Pesquisas Epidemiológicas em Nutrição e Saúde - NUPENS, Universidade de São Paulo.

POPKINS, B. M., 1994. The nutrition transition in lowincome countries: An emerging crisis. Nutrition Reviews, 52:285-298.

RAMOS, L. R.; ROSA, T. E. C.; OLIVEIRA, Z. M.; MEDINA, M. C. G. \& SANTOS, F. R. G., 1993. Perfil do idoso em área metropolitana na região Sudeste do Brasil: Resultados de inquérito domiciliar. Revista de Saúde Pública, 27:87-99.

RIBEIRO, F. S. N., 1994. Da Identidade do Pesquisado à Identidade da Pesquisa: Os Trabalhadores Brasileiros na Pesquisa Nacional sobre Saúde e Nutrição. Dissertação de Mestrado, Rio de Janeiro: Escola Nacional de Saúde Pública, Fundação Oswaldo Cruz.

RISSANEN, A.; HELIÖVAARA, M.; KNEKT, P.; REUNANEN, A.; AROMAA, A. \& MAATELA, J., 1990. Risk of disability and mortality due to overweight in a Finnish population. British Medical Journal, 301: 835-837.

SOLOMONS, N. W., 1992. Nutrition and aging: potentials and problems for research in developing countries. Nutrition Reviews, 50:224-229.

SOLOMONS, N. W. \& MAZARIEGOS, M., 1992. Nutrición, envejecimiento y urbanización. Archivos Latinoamericanos de Nutrición, 42:S84-S86.

STEEN, B., 1988. Body composition and aging. Nutrition Reviews, 46:45-51.

TAYBACK, M.; KUMANYIKA, S. \& CHEE, E., 1990. Body weight as a risk factor in the elderly. Archives of Internal Medicine, 180:1065-1072.

VELLAS, B.; CONCEIÇÃO, J.; LAFONT, C. H.; FONTAN, B.; GARRY, P. J.; ADOUE, D. \& ALBAREDE, J. L., 1990. Malnutrition and falls. Lancet, 336:1447.

VERAS, R. P.; RAMOS, L. R. \& KALACHE, A., 1987. Crescimento da população idosa no Brasil: Transformações e conseqüências na sociedade. Revista de Saúde Pública, 21:225-233.

VERAS, R. P., 1994. País Jovem com Cabelos Brancos: A Saúde do Idoso no Brasil. Rio de Janeiro: Editora Relume-Dumará/Eduerj.

WHO (World Health Organization), 1989. Health of the Elderly. WHO Technical Report Series 779. Geneva: WHO.

WHO (World Health Organization), 1995. Physical Status: The Use and Interpretation of Anthropometry. WHO Technical Report Series 854. Geneva: WHO.

WORLD BANK, 1994. A New Agenda for Women's Health and Nutrition: Development in Practice. Washington, D.C.: World Bank Publications. 